\title{
ARE PULSATIONS A USEFUL PROBE OF THE STRUCTURE OF THE OUTER LAYERS OF WHITE DWARFS $?$
}

\author{
P. Brassard, G. Fontaine, and F. Hesemael \\ Département de Physique, Université de Montréal \\ SD. Kawaler \\ Center for Solar and Space Research, Yale University
}

The most fundamental aspect of white dwarf seismology is the determination of the gravity-mode (g-mode) period structures of models of isolated pulsating white dwarfe. These stars show multiperiodic luminosity variations which result from the superposition of excited pulsation modes. Among the many oscillation modes available in the very rich nonradial g-mode spectra of white dwarfs, the observed modes are selectively chosen by a filtering mechanism. Although the period evolution is strongly tied to the core temperature evolution in a white dworf, the period structure remains largely specified by the mechanical properties of the star. The most basic structural feature of a white dwarf is its highly degenerate interior, which leads to nearly isothermal and nearly isentropic stratifications in the core region containing more than $99 \%$ of the mass of the star. In particular, because the density gradient is almost adiabatic throughout the interior of a white dwarf, the BruntVaisold frequency (see below) is very small there and low-order g-modes connot propagate. As a result, g-modes are essentially envelope modes in white dwarfs, with large amplitudes occuring only in the non-degenerate outer layers. One can thus expect that $g$-modes in white dwarfs are extremely sensitive to envelope properties such as compositional stratification and partial ionization mechanisms. Compositional stratification is, in fact, the second structural feature of a white dwarf model which has strong effects on the period structure. Indeed, trapped modes result when a resonance or near-resonance occurs between the local $g$-mode radial wavelength and the thickness of one of the composition layers. This results in a period structure which strongly bears the signature of compositional stratification in the outer layers. Thus, it has been widely accepted that white dwarf pulsations probe primarily the outer layers of these stars. This point of view has been borne out by detailed pulsation calculations carried out by several independant groups (see Vinget 1987 and references therein). 
A surprisingly different stance has been taken recently by Pesnell (1987; see also Cox et $a$. 1987) who claims that $\mathrm{g}$-modes of white dwarfs probe much deeper than previously thought. Although such modes have generally very small core amplitudes, the point is made that the only appropriate method to describe where the period of a mode is determined is to consult the weight function of the pulsation mode. As an illustrative example, Pesnell (1987) shows the weight function of a particular $g$-mode (noted $g_{1}^{2}$ here, $1=2, k=1$ ) for a DA white dwarf model computed by Tassoul, Fontaine, and Vinget (1988). The model has a total mass of $0.6 \mathrm{M}_{\odot}$. It has a C-rich core surrounded by a He-rich layer itself surrounded by a H-rich layer. The composition transition layers are treated under the assumption of diffusive equilibrium, which, for the particular model of interest, implies that there are some traces of helium extending down to the center of the stor. The effective temperature of the model is $T_{0}-13,969 \mathrm{~K}$, and the hydrogen (helium) layer has a mass equal to $10^{-10}\left(10^{-2}\right)$ times the total mass of the star. The results of Pesnell (1987) indicate, surprisingly, that the period of the $g_{1}^{2}$ made (which is found to be 59.0s) is determined in the central regions of the model; the woight function showing a maximum at about the half-way point in radius. The results are the some for both the Lagrangian pulsation code developed by Pesnell and for an Eulerian version provided that, in the latter case, the square of the Brant-Vaisald frequency is evaluated numerically to take into account the varying chemical composition due to the presence of helium traces in the deep core. Alarmingly, the period of the $g_{1}^{2}$ mode and, more generally, the complete spectrum of the model are found by Pesnell (1987) to be quite different from that of earlier calculations carried out by Winget (1981) using the same model. Pesnell suggests that his taking into account the changing chemical composition in the deep core (ignored in the Winget calculations) makes the difference. If correct, this would mean that the basic period structure cannot be computed with any amount of confidence because it seems so sensitive to the presence of small traces of helium in the core. For simplicity. the model has been computed under the assumption of diffusive equilibrium, but actual timedependent diffusion calculations carried out by Pelletier (1988) show that this assumption breaks down in the very deep core of a white dwarf with an age characteristic of pulsating DA stars. Thus, the helium distribution in the core of a DA model can only be specified by time-dependent calculations and, until detailed results of such calculations exploring a large yolume of parameter space become available, white dwarf seismology would remain next to useless if the interpretation given by Pesnell (1987) is correct. 
Motivated by the past successes of white dwarf seismology based on Eulerian calculations (in porticular the prediction and subsequent discovery of DB variable stars; see Vinget and Fontaine 1982), we have felt that this new concept of period formation in the core of a white dwarf should be carefully examined. He have therefore embarked, with a fresh look, on a detailed investigation of the period structure of the DA model analyzed by Pesnell. In the process, we have discovered a basic shortcoming of the methods used by that author which (1) explains the discrepant results, and (2) has far-reaching implications for the results of his Lagrangian calculations in degenerate stars in general.

The period structure of a stellar model is largely specified by the epatial distribution of the square of the Bront-Vaisald frequency which is defined by

$$
N^{2}=-g\left[\frac{1}{\rho} \frac{d p}{d r}-\frac{1}{r_{p} P} \frac{d P}{d r}\right]
$$

where $g$ is the local gravity, $r$ is the radial coordinate, $p$ is the density, $P$ is the pressure, and $\Gamma_{1}$ is the first adiabatic exponent. In degenerate stars, it is useful (indeed essential in the deep core; see below) to transform equation (1). It can be shown (cf. Brassard et a/. 1988) that $N^{2}$ can be rewritten as

$$
N^{2}=\frac{p g^{2}}{P} \frac{X_{T}}{X_{p}}\left(\nabla_{o d}-\nabla+\left[-\left.\frac{1}{X_{T}} \frac{\partial \ln P}{\partial \ln X}\right|_{\rho, T} \frac{d \ln X}{\operatorname{din} P}\right]\right) \text {, }
$$

where $X_{T}$ and $X_{p}$ are the usual logarithmic pressure derivatives, $\nabla_{\text {ad }}$ is the adiabatic temperature gradient, $\nabla$ is the actual temperature gradient, and $X$ is the mass fraction of one element at a two-ion composition interface. Written in this form, the contribution of the composition transition zones are explicitly included in the term in brackets. This term is always positive and has non-negligible values only in regions where the abundances of two ionic species are comparable, i.e. in the transition zones themselves. These typically cover relatively narrow regions, some two pressure scale heights wide. In particular, the presence of small traces of helium in the deep core of our model cannet affect the values of $\mathrm{N}^{2}$ there. The dominant physical effect is the fact that $\partial \ln P /\left.\partial \ln X\right|_{\rho, T} \rightarrow 0$ in the central regions becouse the pressure of highly degenerate matter for material with $\mu_{0}=2$ is the seme for a given set $(p, T)$, whatever the proportions of the $\mathrm{He} / \mathrm{C}$ mixture. 
We have used the Eulerion code developed by Hansen (see Kawaler, Hansen, and Winget 1985) to analyze the adiabatic period structure of the DA model discussed by Pesnell (1987). The continuous curve in Fig. 1 shows the distribution of $\mathrm{N}^{2}$ in terms of the radius. The small localized structure aroud $r / R \geq 0.93$ is associated with the $\mathrm{He} / \mathrm{C}$ transition zone. A sharp feature, barely visible around $r / R * 0.995$, is associated with $\mathrm{H} / \mathrm{He}$ transition zone located in the outermost layers ( $\left.\log \Delta M / M_{z}-10\right)$. The $N^{2}$ profile is generally quite smooth and any discontinuities or quasi-discontinuities (such as the structures associated with the composition transition zones) are potential features for resonance effects. Our calculations indicate that the period of the $g_{1}^{2}$ mode is 122.88 in reasonable agreement with the older calculations of Winget (1981) which give 142.38, but a far cry from the 59.08 obtained by Pesnell (1987). Note that we recover exact/y the results of Winget (1981) by ignoring the effects of the composition transition layers on the Brunt-Vosailo frequency as was done in that study. Interestingly, however, we can reproduce the results of Pesnell (1987) performing the following experiment: we now calculate $\mathrm{N}^{2}$, not with equation (2), but rather with the numerical derivatives occuring in equation (1). This procedure is implicit in the Lagrangian formulation of Pesnell and was required by him in his Eulerian code in order to recover the results of the Lagrangian calculations.

The dashed curve in Fig. 1 shows the resulting $\mathrm{N}^{2}$ profile for our reference model. As compared to the continuous curve, additional structure and two spurious "convection" zones (negative values of $\mathrm{N}^{2}$ ) have appeared. It is obvious that the $N^{2}$ profile presented by Pesnell (1887) in his Fig. 1 is quite similar to our dashed curve; apparently, the spurious "convection" zones were suppressed by Pesnell. All other features of Pesnell's figure are accurately reproduced, however. As noted before, the quasi-discontinuities that are present in our dashed curve and in the $N^{2}$ profile shown by Pesnell (1987) can isolate and trap certain modes if resonance conditions are met. These modes are trapped in the deep interior, however, implying very large kinetic energies and very low growth rates. More importantly, it is the large systematic differences observed between the dashed and continuous curves in our Fig. 1 that are directly responsible for the large differences found in the period of the same mode. We find, in our altered calculation, a period of 58.78 for the $g_{1}^{2}$ mode, very close to the value of Pesnell (1987) but quite different from our original estimate. The fundamental reason for this discrepancy is that a numerical evaluation of the derivatives appearing in equation (1) encounters serious difficulties 
in degenerate matter. Indeed, with a slight rearrongement of terms, we can write

$$
N^{2}=-\frac{g}{P} \frac{d P}{d r}\left(\frac{d \ln p}{d \ln P}-\left.\frac{\partial \ln p}{\partial \ln P}\right|_{s}\right) \text {. }
$$

where $s$ is the specific entropy per gram. In the nearly adiabatic interior of a degenerate star, the numerical evaluation of derivatives boils down to computing $\mathrm{N}^{2}$ by taking the difference of two nearly equal quantities. By contrast, our formulation of $\mathrm{N}^{2}$ given by equation (2) is reliable everywhere. In the deep core, the term in bracket does not contribute, $\nabla$ is also negligibly small because of the nearly isothermal conditions, $\nabla_{\text {ad }}$ remains a number with typical values slightly under 0.4 , and $N^{2}$ is evaluated by multiplying various quantities. Of course, the two formulations are equivalent in principle, and we indeed observe that the two curves shown in Fig. 1 merge together for large values of $r / R$, i.e. in the outer layers where the degree of degeneracy decreases substantially. Thus, numerical evaluations of derivatives implicit in the Lagrangian formalism of Pesnell lead to unreliably noisy $\mathrm{N}^{2}$ profiles in white dwarf models with concomitant dromatic consequences on the period structure. Not surprisingly, the region of period formation is also affected by these problems. For example, Fig. 2 constrasts the two weight functions which we have computed for the $g_{1}^{2}$ mode of interest. The continuous curve refers to the weight function for the eigenmode computed with our equation (2) for $N^{2}$ and leading to a period of 122.8s. Quite clearly, the mode is an envelope mode. By contrast, the dashed curve, based on the use of equation (1), leads to the conclusion that the period is formed in the deep interior as in Pesnell (1987). Ve have thus clearly identified the origin of the discrepant period and weight function of the eigenmode discussed by Pesnell (1987). We feel that this numerical experiment should put to rest the idea that white dwarf pulsatione probe the deep core.

In summary, we have found evidence that the implicit numerical differencing used in the Lagrangian pulsation code of Pesnell leads to very serious difficulties when used with models of degenerate stars. These difficulties are at the origin of his suggestion (Pesnell 1987) that white dwarf periods are formed in the deep interior. We reaffirm the prior results of other investigations; g-mode pulsations in white dwarfs are truly envelope modes. The implications of our findings on the work of Cox et a/. (1987) need to be carefully evaluated. In particular, the besic pertod structure of their models (i.e. the most fundamental aspect of asteroseismology) is 
clearly open to question. Because of this, their nonedtebetlc results concerning primarily driving and damping must be considered premature; thus, their conclusions about the complete insensitivity of the $Z Z$ Ceti theoretical blue edge temperature to the hydrogen loyer mass remain clearly questionable.

We are grateful to DE. Winget for useful discussions. This work was supported in part by the NSERC Canada, by the fund FCAR (Quábec), and by a E.HR. Steacie Fellowship to one of us (GF).

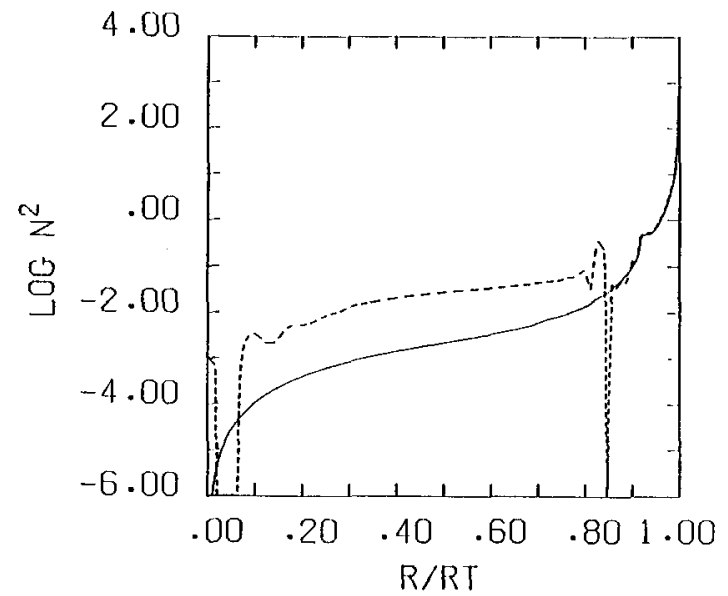

7.5. 1 - The square of the Erunt-Vaisallib frequency as a function of rodius for our DA white dworf modol. The continuous arve corresponds to the computations based on equation (2): the dashed arve to those based on equation (1) with numerical derivatives.

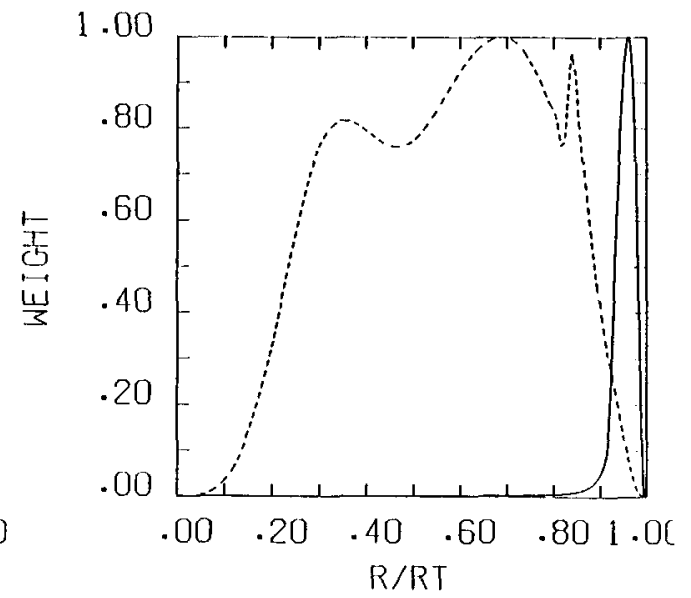

My. 2 - The weight function of the $g_{1}^{2}$ mode in terme of the rodius. The continuoue and dasted arves correspond to the two sets of colculations referred to in the previous coption

\section{REFRaNCES}

Erassord, P., Fontains, G., Wesemoel, F., Tassoul, M., and Kowaler, 5.D. 1989, in preparation.

Cox, AN., Starrfield, S.G., Kidman, RB., and Poonall, WD. 1987, Astrqahys. J., 317, 303.

Kawaler, S.D., Harsen, C.J., and Wingot, DE. 1985, Astroohys. J, 248, 547.

Pelletier, C. 1909, private communication.

Peonell, WD. 1987, in Stelfar Putsation: A Memorial to John P. Cox, AN. Cox, WM. Sparks, and S.G. Stcrrfield ods., Springer-Verlog, Berlin, p. 363.

Towsoul, M., Fontaine, G., and Winget, DE. 1989, in preparation.

Winget, DE. 1981, Ph. D. thesis, University of Rochester.

Winget, DE. 1987, in Hoskghts of Astronomy, JP. Swings ed., p. 221.

Winget, DE., and Fontaine, G. 1982, in Pusetions in Classical and Cataclyswic Vartable Stars, JP. Cox and C.J. Hansen eds., Uhiversity of Colorado, Boulder, p. 46. 